\title{
Virtual Reality platform for functional magnetic resonance imaging in ecologically valid conditions
}

\author{
Baptiste Gauthier \\ Laboratory of Cognitive \\ Neuroscience, Center for \\ Neuroprosthetics and Brain Mind \\ Institute, Swiss Federal Institute of \\ Technology (EPFL), 9 Chemin des \\ Mines, 1202 Geneva, Switzerland \\ baptiste.gauthier@epfl.ch
}

\author{
Louis Albert \\ Laboratory of Cognitive \\ Neuroscience, Center for \\ Neuroprosthetics and Brain Mind \\ Institute, Swiss Federal Institute of \\ Technology (EPFL), 9 Chemin des \\ Mines, 1202 Geneva, Switzerland \\ louis.albert@epfl.ch
}

\author{
Roberto Martuzzi \\ Fondation Campus Biotech Geneva, 9 \\ Chemin des Mines, 1202 Geneva, \\ Switzerland \\ roberto.martuzzi@fcbg.ch
}

\author{
Bruno Herbelin \\ Laboratory of Cognitive \\ Neuroscience, Center for \\ Neuroprosthetics and Brain Mind \\ Institute, Swiss Federal Institute of \\ Technology (EPFL), 9 Chemin des \\ Mines, 1202 Geneva, Switzerland \\ bruno.herbelin@epfl.ch
}

\begin{abstract}
Functional magnetic resonance Brain Imaging (fMRI) is a key noninvasive imaging technique for the study of human brain activity. Its millimetric spatial resolution is at the cost of several constraints: participants must remain static and experience artificial stimuli, making it difficult to generalize neuroscientific results to naturalistic and ecological conditions. Immersive Virtual Reality (VR) provides alternatives to such stimuli through simulation, but still requires an active first-person exploration of the environment to evoke a strong sense of presence in the virtual environment. Here, we report how to compensate for the inability to freely move in VR by leveraging on principles of embodiment for a virtual avatar, to eventually evoke a strong sense of presence with a minimal motion of the participant. We validated the functionality of the platform in a study where healthy participants performed several basic research tasks in an MR-specific immersive virtual environment. Our results show that our approach can lead to high sense of presence, strong body ownership, and sense of agency for a virtual avatar, with low movement-related MRI artifacts. Moreover, to exemplify the

Baptiste Gauthier and Louis Albert contributed equally as first authors. Bruno Herbelin and Olaf Blanke contributed equally as last authors.

Permission to make digital or hard copies of all or part of this work for personal or classroom use is granted without fee provided that copies are not made or distributed for profit or commercial advantage and that copies bear this notice and the full citation on the first page. Copyrights for components of this work owned by others than ACM must be honored. Abstracting with credit is permitted. To copy otherwise, or republish, to post on servers or to redistribute to lists, requires prior specific permission and/or a fee. Request permissions from permissions@acm.org.

VRST '21, December 08-10, 2021, Osaka, Japan

(C) 2021 Association for Computing Machinery.

ACM ISBN 978-1-4503-8557-2/21/10 . .\$15.00

https://doi.org/10.1145/3466752.3480085
\end{abstract}

\author{
Olaf Blanke \\ Laboratory of Cognitive \\ Neuroscience, Center for \\ Neuroprosthetics and Brain Mind \\ Institute, Swiss Federal Institute of \\ Technology (EPFL), 9 Chemin des \\ Mines, 1202 Geneva, Switzerland; \\ Department of Neurology, University \\ of Geneva, 24 rue Micheli-du-Crest, \\ 1211 Geneva, Switzerland \\ olaf.blanke@epfl.ch
}

versatility of the platform, we reproduced several behavioral and fMRI results in the perceptual, motor, and cognitive domains. We discuss how to leverage such technology for neuroscience research and provide recommendations on efficient ways to implement and develop it successfully.

\section{CCS CONCEPTS}

- Human-centered computing $\rightarrow$ Human computer interaction (HCI); Interaction paradigms; Virtual reality; Human computer interaction (HCI); $\mathrm{HCI}$ design and evaluation methods; Laboratory experiments.

\section{KEYWORDS}

Immersive Virtual Reality, Ecological, Presence, fMRI

ACM Reference Format:

Baptiste Gauthier, Louis Albert, Roberto Martuzzi, Bruno Herbelin, and Olaf Blanke. 2021. Virtual Reality platform for functional magnetic resonance imaging in ecologically valid conditions. In 27th ACM Symposium on Virtual Reality Software and Technology (VRST '21), December 08-10, 2021, Osaka, Japan. ACM, New York, NY, USA, 12 pages. https://doi.org/10.1145/3466752. 3480085

\section{INTRODUCTION}

\subsection{Magnetic resonance in human neuroscience}

Functional magnetic resonance imaging (fMRI) is the leading research tool to investigate the human brain. fMRI allows measuring brain activity non-invasively in response to a large variety of sensory stimuli, for actions and movement execution, as well as 
higher-level brain processes [10, 11, 25]. Therefore, fMRI has enabled major advances in the understanding of perceptual, motor, cognitive, social, and affective brain mechanisms [28, 36]. The success of fMRI also comes from logistic advantages: MR scanners are routinely used in clinical and research settings and are thus widely available. Such technology can offer stimulation (input) and response (output) devices compatible with those classically used in experimental psychology and human neuroscience (e.g., flat screen, headphones, and button-press devices). Yet, the utilization of fMRI in neuroscience research is facing major challenges regarding the ecological properties of the stimulation. The primary limiting factor comes from the physical constraints imposed on the participant's body posture and position. In the MR magnet, the person is in a supine position with the head fixed inside a head coil, and the head and body must remain static to avoid head movement-related artifacts in fMRI data. This immobility and constrained posture strongly limit the possible interactions that participants can carry out during a given task and are constant reminders for participants that they are located inside the MRI magnet. Another major limiting factor is technological: standard electronic devices cannot be used as the MR magnet exert both a static (preventing a reliable use inside the magnet) and a dynamic magnetic field during image acquisition (prone to interfere with most electronic devices not specially crafted for the MR environment). The limited availability of MRI-compatible hardware, combined with the very loud noise of all MRI systems (interfering with auditory stimuli even when combined with the systematic use of headphones and noise-canceling earplugs) are thus serious constraints for the implementation of testing environments.

As a consequence, fMRI has been mainly used for imaging specific tasks involving stimuli isolated from their natural context of exposure (for instance, contrasting pictures of static faces without bodies with pictures of houses [23]). This was originally compatible with the scientific program of human neuroscience based on the experimental decomposition of complex neural and cognitive processes into simpler components, and thus simpler artificial stimuli, in order to study them separately [7].

\subsection{Virtual reality for cognitive neuroscience}

Virtual Reality (VR) technologies offer promising approaches to pally to the lack of naturalistic testing environments in human neuroscience. As outlined by de Gelder [13], the classical research methodology consisting in using controlled but simplified stimuli are today superseded by immersive VR approaches in which major parts of the perceptual environment are replaced, thus allowing studying perception, actions, and higher cognition in a controlled yet rich and interactive environment. The immersive capabilities of VR technologies, defined as their ability to deliver a surrounding and convincing environment to participants [39], provide experimental psychology and neuroscience with unprecedented possibilities of realistic stimuli. For instance, instead of hearing a sound or looking at a video clip of a person talking, participants would see a 3D character talking, moving in a room while his voice is spatialized. Fundamentally, VR is based on the ability of a simulation to provide participants with the subjective experience of being located in a virtual place instead of the real world (place Illusion [42]) and with the feeling of embodying a virtual body in place of the physical body (sense of embodiment [21]). Extending on these principles, VR then opens the possibility for cognitive neuroscience research to manipulate the congruence of sight, sound, and touch to manipulate and to control a large spectrum of experiential illusions, i.e. perceptions and feelings that are deviating from the information coming from the senses [20,44]. For instance, the so-called body-swap illusion [35] recently allowed to understand how body ownership interferes with semantic self-representation [48].

Immersive VR is today most widely implemented using headsetVR setups [46]. Using head-mounted displays (HMD) to track the participant's head motion in space, the system presents visual stimuli at a high refresh rate to match the virtual viewpoint according to the participant's head position and orientation, thus providing a first-person view as if the participant was part of the scene. This process is essential for supporting the natural sensorimotor contingencies involved during the active exploration of the scene, which are key to provide the subjective experience of 'being there' in the virtual world [43], and to provide the ability to 'do' there [39]. Recent developments in VR have thus demonstrated the importance of providing users with an avatar impersonating them. Interestingly, key insights into our understanding of the phenomenon of virtual embodiment [27] and to its actual definition [21] have come from research in cognitive neuroscience, specifically on the sense of agency and self-consciousness. A large variety of studies have since demonstrate how the embodiment illusion allows participants to be someone else in VR [33], become a child [3], change the color of their skin [26], or even have an extra limb [47]. Extending the former understanding of presence in VR, embodiment is now acknowledged as a constitutive part of the overall VR experience [16], and several studies have demonstrated its beneficial impact on e.g. immersion and emotional response [51] or on episodic memory in VR [12]. Conversely, disruptions of embodiment have also been shown to break presence and immersion, such as upon the occurrence of visuo-tactile conflicts [6] or loss of agency [37].

\subsection{Contributions: MRI-compatible immersive headset-VR platform}

Our study contributes to the use of immersive VR in neuroimaging in two key aspects. First, we tailored an immersive VR platform specifically for the MRI environment and demonstrate that we successfully achieve high levels of presence and embodiment while simultaneously controlling for possible decrease of MR data quality by motion-induced artifacts. Specifically, we mitigate some of the perceptual consequences of both immobility and supine position constraints imposed by the MRI on participants by leveraging the ability of VR to provide participants with the subjective experience of presence in a virtual laboratory. To this aim, we introduce an original methodology for merging the physical and the virtual worlds. Following this procedure, participants experience being in a virtual examination room in a first-person embodied perspective, thus helping them to comply with the limitations of head and body movement needed for imaging while opening the possibility to bring virtual objects, characters, and interactions in the virtual environment in an ecological fashion. Overall, we address the technological constraints linked to the use of VR equipment in MRI 
by merging commercially available MR-compatible products into a single, integrated solution. Essentially, visual immersion is now possible with recent MR-compatible stereoscopic goggles providing sufficient spatial resolution, fields of view, and optical motion capture solutions for MRI, enabling the tracking of participants for embodiment and interaction.

Second, the results from our pilot validation study demonstrate that we can both replicate classical fMRI research paradigms for perceptual, motor, and cognitive tasks as well as make them more ecological. This efficiency further leverages our approach over the prevailing use of 3D-desktop gaming technologies in neuroscience research (e.g., screen with joystick control). Taken together, these methodological and technological advances provide a way to replace experimental setups with virtual ones, thus offering the possibility to implement complex and natural experimental tasks in VR while the participant is inside the MR scanner.

\section{PLATFORM DESCRIPTION}

\subsection{Building principle}

Our VR platform for human neuroscience research and fMRI neuroimaging is built around the principle of scalability and the possibility of improving individual elements of the platform. Its main components requirements are MR-compatible stereoscopic googles with high resolution and large field of view to maximize immersion and presence, a motion-tracking system to support sensorimotor contingencies in the virtual environment, a virtual model of the physical room to merge the physical and virtual worlds, and a "VR ready" computer to run the simulation at a high frame rate.

\subsection{Equipment}

To acquire brain images, we used a 3T Siemens Magnetom Prisma MRI scanner (Siemens

Healthcare Gmbh, Erlangen, Germany) equipped with a 64channel head-and-neck receiving coil. To display visual stimuli to participants, we used the Visual System HD (NordicNeuroLab, Bergen, Norway) with integrated eye-tracking. It was composed of two full-HD resolution displays (1920 x 1200, 16:10 WUXGA) allowing $3 \mathrm{D} /$ stereoscopic rendering at $60 \mathrm{~Hz}$ with video input from two HDMI cables. The display provided a diagonal field of view of $60^{\circ}$ (52.1 $1^{\circ}$ horizontal, $34^{\circ}$ vertical). In the simulation, the virtual cameras were placed at the location of the avatar's eyes, rendering a perspective projection of the world with a corresponding $52.1^{\circ}$ horizontal field of view.To acquire the motion of moving objects and of the participant's different body parts in an online fashion in the scanner, we highly recommend a six degree of freedom (6 DOF) motion capture system updating at an equal or faster rate than the display refresh rate and placed inside the MRI room. To this end, we used a Qualisys MRI-compatible motion capture system composed of six Qualisys Oqus 500+m cameras (180 Hz, 4 MegaPixel resolution). Cameras were fixed to the ceiling inside the MRI room to avoid involuntary motion of the camera and avoid optical artifacts due to the window pane between the control room and the MRI room.

A customized handheld allowing hand movement tracking and behavioral response recording using custom buttons were wired to a microcontroller (Arduino Micro) placed inside the scanner room and were as far as possible from the isocenter of the scanner. They were connected to the main computer via a USB optical switch. The microcontroller emulated a keyboard, and 3 buttons were mapped to simulate different keyboard inputs. To record our participants' responses, two hand-held response devices were combined with 6-DOF motion capture trackers. Our homemade response devices were composed of tennis balls (one per hand) with support for motion capture trackers attached to the ball (Figure 1).

In the control room of the MRI, the stimulation computer was connected to the visual, motion tracking, and trigger systems. Our stimulation computer runs on Windows 10 Pro 64 bits and was equipped with Intel Xeon Silver 4110 CPU, NVidia Quadro P6000 Graphics Processing Unit, and 64 GB of RAM. We used a triggering system (Current Designs 932; Current Designs Inc., Philadelphia, PA) to synchronize the MRI data acquisition and the simulation software.

\subsection{System setup}

The headset (VisualSystem HD) was mounted on the MRI headand-neck coil with a coil-specific adapter. An adjustable arm between the coil-specific adapter and the VisualSystem HD allowed fast positioning to the preferred angle of view. To ensure the synchronization between the two screens and facilitate the use of the googles, we used the Nvidia multi-screen technology (Nvidia Mosaic software solution) to create from these two screens a single screen of resolution $3840 \times 1200$ (render for eye place side-by-side). The VisualSystem HD was also equipped with a built-in diopter correction $(-7$ to +5$)$ and pupil distance adjustment (44 to $75 \mathrm{~mm}$ ) that facilitated the calibration for each participant.

The Qualisys Motion Capture system provides solutions for connecting multiple cameras, for adjusting cameras aperture, exposure, and marker intensity threshold detection, as well as for calibrating calibrate the system for 3D tracking. In the present implementation, two rigid bodies (RB) were used to track participants' hands' position and rotation, two others to track participants' legs movements, and a fifth RB was tied to the MRI table to track its displacement during entrance and exit in and out the MR magnet. The mapping of hands' position was processed in reference to the MRI table; the position of the hand-held RBs was mapped so that the participant's hand projection on a 2D plane normal to the MRI table matches the virtual avatar's hand projection on a $2 \mathrm{D}$ plane normal to the virtual MRI table. This solution ensures that all participants were able to interact with virtual elements without the need for the calibration of the forearm's length, while correctly matching real and virtual hand collisions with the MRI tube. The leg RBs were attached pointing upward on top of participants' knees (one on each knee), and their yaw rotation was mapped to the virtual avatar knees rotation.

The virtual environment displayed through the VR engine (Unity 3D) was a replication of the real MRI room, with realistic illumination and shading. Particular care was given to details in the parts of the virtual room visible from the participant's point of view (Figure 1). Virtual objects, such as a TV screen, were placed in the scene for the purpose of the experiment.

\section{PROCEDURE}

The general procedure for conducting experiments in this VR-MRI platform is centered around a core experimental phase. Testing, 

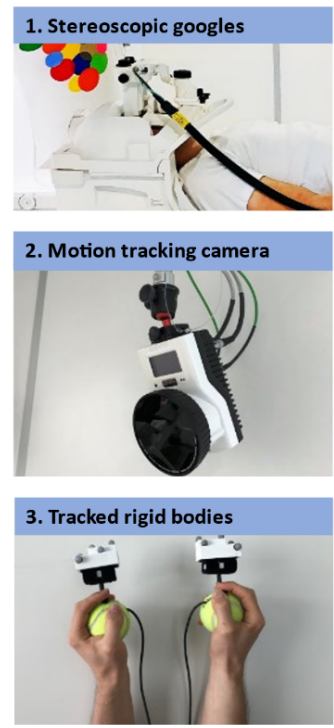
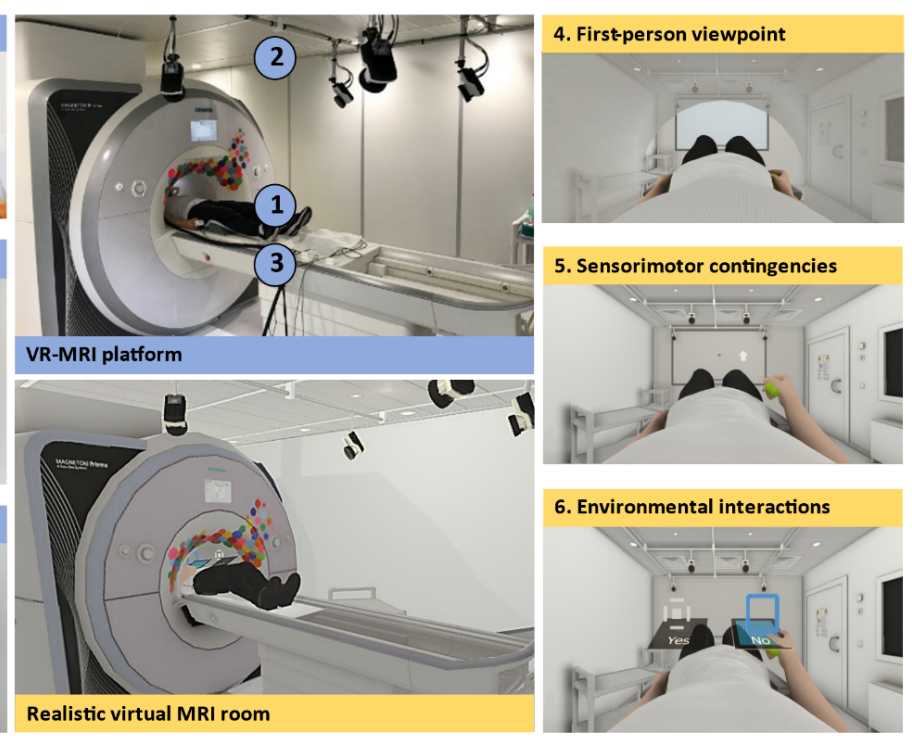

5. Sensorimotor contingencies

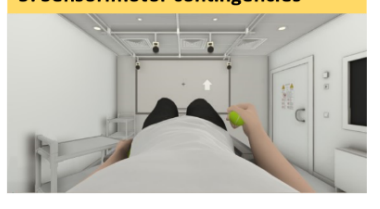

6. Environmental interactions

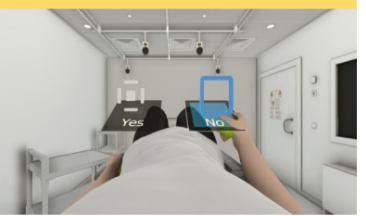

Figure 1: The VR-MRI setup relies on 3 main devices: (1) a pair of stereoscopic googles displaying the virtual environment, (2) six motion-tracking cameras, and (3) two rigid bodies for 6-DOF tracking combined with custom response devices held by the participants. The virtual scanner was a carefully design environment with the best visual matching possible to enable immersion. After entry in the MR magnet-done synchronously with the entry in the virtual scanner-the main ecological features of the environment are enabled: (4) first-person viewpoint from the position of a shape-matched virtual body, (5) the sensorimotor contingencies with virtual upper limb movements following those of the real upper limbs, and (6) the environmental interactions allowed with reactive virtual objects.

preparation, and familiarization phases ensure that participants are fully immersed for the whole duration of the experimental tasks, which are conducted entirely in VR (Figure 2). The procedure ends with a specially designed exit phase in VR before the experimenter removes the VR equipment.

\subsection{Participant preparation}

The participant was then placed in a supine position on the MR bed. The visual display was fixed onto the head-and-neck coil so that the googles were stable with respect to the head. To ensure a correction vision, the participant manually adjusted the interpupillary distance and diopter correction for each eye while a fixation cross was displayed on the headset. Some pictures from the stereoscopic acuity test with low disparity were also displayed to fine-tune diopter correction and ensure the participant perceived depth in the MRI googles.

\subsection{Virtual avatar preparation}

To match the participant's actual and virtual appearance, they were asked to wear dark pants, a white t-shirt, and black shoes (all provided). We then measured their waist size (width and height at the level of the belly button). To provide a reasonably naturalistic experience of the virtual body, our framework allowed multiple modifications to match the virtual avatar as much as possible with the participant's appearance. First, we selected the gender of the avatar (male or female). Second, we matched the skin color of the avatar with the skin color of the participant. And third, we scaled the virtual avatar waist to have similar proportions and adjusted hips and chest size accordingly.

\subsection{First immersion and familiarization with the virtual environment}

In order to transit from the real to the virtual world and enhance the presence in the virtual world, 3D visuals of the replicated MRI room were shown in the stereoscopic googles, matching what the participants would see from their position while lying on the MR bed (i.e., looking at the ceiling). This gave the impression of looking at the actual MRI room, but through optics.

Then, during the entry of the participant into the scanner, the movement of the virtual viewpoint followed the movement of the MRI table as it entered the magnet (tracking of the MRI table). Importantly, during the entry trajectory, the viewpoint progressively rotated from looking up at the ceiling toward looking down at the feet (almost horizontally), with a total rotation of 76 degrees. This provided participants with a first-person view corresponding to a person supine on the back but inclined forward and looking at her/his feet while still being in supine position. After the entry phase, we asked participants to carry out selected movements of 


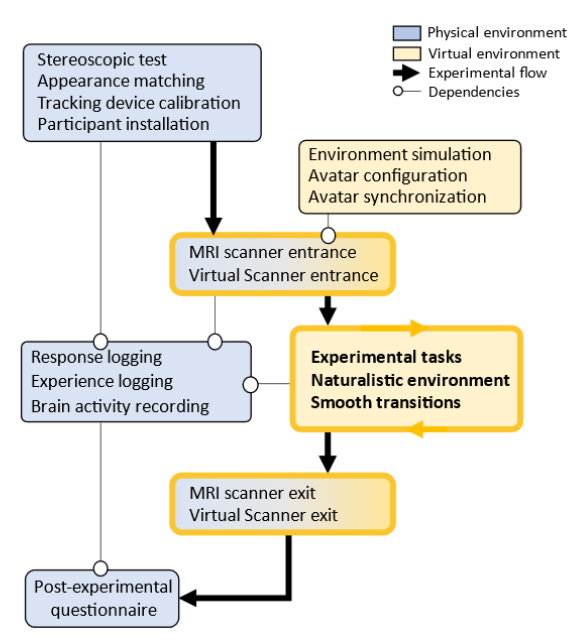

Figure 2: Procedure Overview. Each step is part of the physical setup (blue) or of the virtual setup (orange). Two key steps are the entrance and exit, as they require a synchronous motion combined to a virtual viewpoint shift to be able to provide an effective, ecologically valid virtual environment. Experimental conditions are performed when the transfer into virtual reality is enabled, and separate runs are separated by designed virtual transitions.

their knees and of their hands while holding tennis balls. We then asked them to simply observe how their virtual body moves based on those tracked and animated movements in VR (replicating the response devices). During this familiarization phase, we designed the MR magnet as shown in VR to slowly fade out and become invisible, so that eventually participants would see the scanner room, but without the scanner.

Through this progressive familiarization, participants maintained a strong sense of being located in the scene and eventually perceived being lying on a bed in an open examination room. These steps enabled a plausible, near-ecological experience of the virtual environment with a large space available around them for stimuli presentation and interaction.

\subsection{Further transitions}

To maintain ecological conditions, the transition between instructions, experimental conditions, and questionnaires should avoid interrupting immersion. To ensure as smooth a transition as possible, we created a routine for switching from one experimental block to another, as described hereafter. Text instructions were displayed on the virtual screen. At the end of an experimental block, text instructions (5 seconds) informed the participant that the block was finished and that they could relax and shortly close their eyes if they wish. A few minutes after, text instructions informed that the experiment will soon start again (5 seconds). Then, the text instructions faded to a fully black screen ( 3 seconds) and turned to the virtual display progressively back on (3 seconds), to avoid obvious "pop-outs" of scene changes between the previous and the following stimuli.
We also implemented a system of checkpoints at each transition period between blocks, allowing the experimenter to bring the participant back to a previous point of the experimental flow in case of unexpected problems. For fMRI acquisition, any unexpected problem causes to stop the acquisition and restart the entire acquisition block, thus a checkpoint system is essential. Going back to a previous checkpoint triggered automatically the visual transition described above, preserving immersion even facing unplanned events. We use visual fading as that kind of transition appears smoother than simple cuts (i.e., abrupt transition from one frame to another) and less prone to trigger breaks in presence [18, 31]. When the experiment was finished, the exit from the virtual environment consisted in the reverse of the scanner entrance. From the $76^{\circ}$ tilted viewpoint inside the virtual scanner, the participant's viewpoint moved progressively toward the exit position in a supine viewpoint (looking at the ceiling) while physically moving toward the initial position onto the MRI table (outside the MRI bore).

\subsection{Experimental tasks}

All experimental tasks were conducted entirely in VR. In the virtual environment, visual stimuli were displayed according to the needs of each task: either on the virtual TV screen (in VR) shown as hanging on the wall of the virtual room or as virtual objects in the scene. All experimental conditions were performed while participants saw and controlled their avatar from a first-person perspective.

Many neuroscience-relevant tasks can thus be performed in this environment, with great freedom to integrate virtual experimental setups, either replicating classical on-screen laboratory systems or simulating an ecological situation. To test and demonstrate the VR-MRI platform, we focused on a perceptual (random dot kinematogram - RDK, passive viewing), a motor (arm lifting task - MVT), a cognitive (own-body transformation task - OBT), and a bodily self-consciousness task (BSC) (Figure 3). Among those tasks, eight runs of the OBT task were pseudo-randomly alternated, followed by the RDK and finally the MVT task, always in this order. The switch between tasks is self-paced by participants by confirming the instructions and always separated by an additional 5-second pause.

3.5.1 Perceptual task. Classical visual tasks usually display stimuli on a computer screen. This was replicated with the virtual TV screen. As the perception of visual motion represents an important ability of our visual system in a dynamically changing environment, we tested a random-dot kinematograms passive viewing task, known to induce apparent motion perception [14]. The virtual TV screen, placed in front of participants in first-person view, was used to reproduce the visual laboratory computerized task. Participants were instructed to fixate a central cross while seeing a group of white dots on a black background (3). Coherent motion either moved from left to right, from right to left or was stationary. Each visual stimulus lasted 3 seconds and was followed by a 5 -second baseline gray screen with a black central fixation cross. The full block consisted of a random alternation of 5 left to right, 5 right to left, and 5 static stimuli (not used). We obtained brain activity maps by contrasting left to right from right to left movements. 

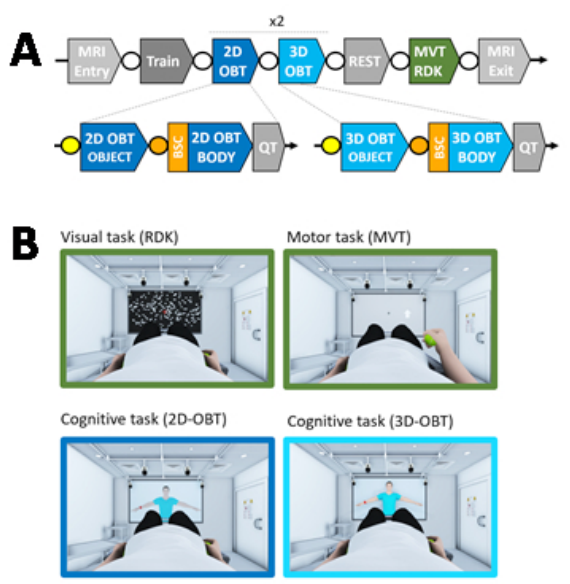

Cognitive task (3D-OBT)
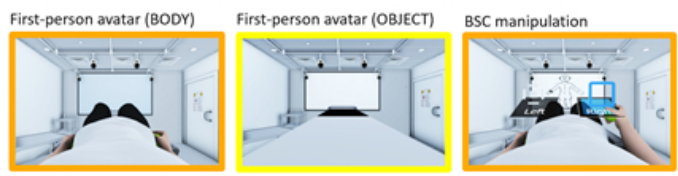

Figure 3: Experimental design (A) and Snapshots of the virtual environment during the different phases of the experiment (B). Following the virtual MRI entry phase, a training phase during which the MRI magnet faded consist of several runs of OBT tasks. Two repetitions of $2 \mathrm{D}$ - and $3 \mathrm{D}-\mathrm{OBT}$, both with BODY and OBJECT first-person avatars followed. After a resting session and the motor (MVT) and perceptual (RDK) tasks, the experiment ended with the virtual MRI exit. A checkpoint (circles) preceded each phase. A smooth visual transition preceded each change from BODY to OBJECT and vice versa. One BSC induction phase preceded BODY blocks.

3.5.2 Motor task. In human neuroscience, we often use motor actions using upper limbs to acquire responses from participants and measure corresponding reaction times. In the present virtual environment, as the arm and knees are tracked, we could also faithfully transfer the movement of these body parts on the virtual avatar. As participants were lying in a supine position, the avatar's full-body representation provided a realistic and synchronous visual feedback of the participant's real movement, enabling the measure of sensorimotor integration during motor actions (Figure 3).

At first, an upward-pointing arrow was displayed on the virtual screen ( 0.5 seconds) followed by a baseline consisting in a fixation cross on gray background ( 5 seconds). During the block, the participant had to lift the arm corresponding to the side where the arrow was displayed and return it to the starting position. The avatar's arm was lifted synchronously with the participants' movement. The experiment consisted of a random alternation of 10 elevations of the left arm and 10 of the right arm. We obtained brain activity maps by contrasting right arm versus left arm elevations.

3.5.3 Cognitive task. On-screen display of stimuli for cognitive neuroscience is pervasive. However, this approach may miss aspects related to the natural, ecological unfolding and integration of the cognitive processes as they happen in real life. Thus, in this pilot, we specifically looked for an advantageous replacement of a bidimensional (2D) stimulus by a 3D object co-located in the virtual environment. To this end, we selected a well-known mental rotation task: the own-body transformation (OBT) task [52], which requires the mental rotation of a displayed character.

In our implementations of the OBT task, participants had to judge if a character wore an indicated bracelet on the left or the right hand. The character could be in a front- or back-facing position $[4,52]$. To provide judgments, participants had to press a button with their left or right hand for an indicated left- and right-hand bracelet, respectively (Figure 3). Two versions of the OBT task were implemented: the character could either be, as in the original study [52], a 2D flat picture of a character displayed on the virtual screen (2D-OBT) or, for our comparison, a 3D virtual character standing in front of the virtual screen (3D-OBT). We obtain brain activity maps for evaluating the cognitive tasks by contrasting OBT blocks to resting baseline.

Furthermore, one interesting possibility allowed by our VR-MRI platform is the ability to provide multisensory cues congruent with a natural first-person perspective: the body is seen close to the actual body position, the limbs are moving according to the ones of the real body, and the movements in the virtual environment trigger interactions with virtual objects in a continuous, online fashion, to reproduce the natural sensorimotor contingencies [43] and satisfying the conditions for embodiment (self-location, body ownership, and agency [21]). Therefore, we tested the effect of sensorimotor stimulation, known to manipulate bodily self-consciousness, for the avatar during the OBT task (BSC task). Participants were presented either with a color-matched rectangular box at the place of their body in first-person view (OBJECT condition, control) or with a body-matched avatar (BODY condition). To ensure embodiment in this condition, we added an additional sensorimotor induction phase of self-identification to the first-person viewed virtual avatar (BSC) before each BODY condition of the OBT task. This phase was composed of 12 OBT trials (simplified 2D figures on the screen) and the participant had to answer by raising the left or right hand to activate virtual buttons in front of them (Figure 3). This induction phase thus served as training for the experimental task and, importantly, provided a direct interaction between the virtual body and the virtual environment to strengthen embodiment. Additional brain activity maps evaluating embodiment were obtained by contrasting BSC phase with OBT blocks.

Each combination of factors (BODY 2D-OBT, OBJECT 2D-OBT, BODY 3D-OBT, and OBJECT 3D-OBT) was acquired twice, leading to 8 blocks in total. The order was pseudo-randomized by shuffling block couples formed by a systematic succession of an OBJECTOBT and the corresponding BODY-OBT (either couple of 2D- or 3D-OBT), in this order. Each block comprised a random alternation of 32 OBT trials for 8 repetitions of front-facing left bracelets, backfacing left bracelets, front-facing right bracelets and back-facing right bracelet characters.

To evaluate the embodiment manipulation at the end of OBT blocks, we presented 5 questions investigating the ownership of the virtual body or object, the agency of the movement, self-location, experienced direction of the point of view, and a control question for suggestibility (Q1 to Q5, respectively) [2, 20, 24]. Participants 
answered questions according to the instructions by pressing buttons of the custom response device. We implemented a continuous scale ranging from "strongly disagree" to "strongly agree" with a self-moving cursor that the participant could stop at the desired position by a button press.

\subsection{Post-experiment debriefing}

After the experiment, a post-scan questionnaire inspired from [49] was administered on paper where participants had to pick a binary choice to answer questions on their subjective sense of presence and agency during the experiment, and about the head tilt during the entry and exit phases. We expected high sense of presence and agency, and that the head tilt would remain unnoticed.

\subsection{Participants}

Eleven right-handed participants with normal vision and no history of neurological or psychiatric disorders were recruited for the study All participants were compensated for their participation and provided informed written consent in accordance with the local Ethical Committee and the declaration of Helsinki (2013). Participants were screened for compliance with high magnetic field safety. To avoid perceptual discrepancies in our results, we excluded from the study one participant identified as 3D-stereo blind.

\section{RESULTS}

\subsection{Perceptual task}

Apparent-motion perception induced by RDK is known to activate visual regions such as hMT+/V5 area [14]. Using the present method, we detected significant activation of hMT+/V5 by contrasting passive viewing of left-oriented and right-oriented RDKs ( $p$ $<0.001$ uncorrected, Figure 4), showing that a classical perceptual task result can be reproduced in our VR-MRI platform using the virtual screen setup.

\subsection{Motor task}

Motor actions engage body-part specific regions of the primary motor cortex and here we choose to record brain activity corresponding to the movement of the left and right arm. We report activation in the right and left dorsal regions of the motor cortex (Area M1) when contrasting left with right movements ( $p<0.001$ uncorrected, Figure 4). This shows that execution of a motor task with feedback results in M1 activation using our VR-MRI platform. Most notably, no significant motion-induced noise artifacts were observed in the MRI signal due to the head motion conveyed by arm movements (see MRI motion evaluation section).

\subsection{Cognitive task}

To analyze participants' behavior, we used a mixed model with participant as a random factor and character angle (front/back-facing), character implementation (2D/3D-OBT), and first-person avatar (BODY/OBJECT) as fixed factors. We found significantly longer reaction times for the OBT task for the front-facing as compared to the back-facing condition ( $p=0.002)$. Reaction times were also longer for the 2D-OBT as compared to 3D-OBT $(\mathrm{p}=0.019)$ and tended to be longer in BODY as compared to the OBJECT condition (not significant, $\mathrm{p}=0.312$ ) (Figure 4). As such, we replicate the classical OBT findings but also show a decrease of the OBT effect when using a $3 \mathrm{D}$ as compared to $2 \mathrm{D}$ character, exposing potential effects of interest using more ecological stimuli (here a co-located virtual character).

Our neuroimaging results show activations for the OBT task in bilateral temporoparietal junctions, premotor and motor cortices, extrastriate body area $(\mathrm{p}<0.001$ uncorrected) as previously found for OBT task $[1,4,40]$ (Figure 4). This replicates classical neuroimaging results of the OBT task using an immersive VR version of the task. Moreover, we found activity in occipital, parietal, and motor regions during BSC manipulation as compared to OBT task, a brain network previously identified as involved in bodily aspects of selfconsciousness [5] (Figure 4).

\subsection{In-scanner questions}

We normalized the subjective ratings per participant by subtracting the control question Q5 from all questions. We found significantly higher BSC ratings of body ownership and agency as compared to OBJECT conditions (linear mixed model with fixed effect of virtual body versus virtual object and random effect of participant, Q1: $t=$ $4.69, \mathrm{p}=0.0014 ; \mathrm{Q} 2: \mathrm{t}=3.38, \mathrm{p}=0.003$; Figure 5).

\subsection{Post-experimental questionnaires}

We overall found a qualitatively high Presence, Agency and low head tilt noticeability (Figure 5), suggesting high levels of immersion using the platform.

\subsection{MRI motion evaluation}

Head motion during fMRI acquisition leads to a decreased signalto-noise ratio of the fMRI signal. In order to quantify the motion during the fMRI acquisition, we used movement metrics as used in Power et al [38] and quantified the sum of absolute translational, rotational, and total framewise displacement (AbsTD, ABsRD, and FWD, in mm, respectively; Figure 6). The absolute translational displacement is the sum of the absolute values of the $\mathrm{X}, \mathrm{Y}$, and $\mathrm{Z}$ translation estimates for a given frame. The rotational version is the sum of the absolute values of the displacements in pitch, yaw, and roll. Framewise displacement is the sum of the absolute values of the differentiated realignment estimates by backward differences for each temporal frame (i.e., each acquired MRI volume). We found on average ( \pm standard deviation) $0.53 \pm 0.37 \mathrm{~mm}, 0.59 \pm 0.45 \mathrm{~mm}$, and $0.27 \pm 0.18 \mathrm{~mm}$ of average translational, average rotational, and framewise displacement. These values correspond to low to moderate noise level, being less than one voxel size (here $2 \mathrm{~mm}$ isotropic).

To quantify the consequence on framewise data rejection-thus accounting for motion peaks-we determined the percentage of frames rejected in a motion censoring strategy (i.e., mask fMRI volume with above-threshold motion level from the statistical analysis) with framewise displacement thresholds: above $0.5 \mathrm{~mm}$ (FDWR05) and above $1 \mathrm{~mm}$ (FDWR1), considered stringent and liberal thresholds for task-based fMRI, respectively [41]. Using 0.5- and 1-mm thresholds, we removed that $9.6 \pm 12.9 \%$ and $3.8 \pm 5.8$ of the frames (Figure 6). Removing $10 \%$ of the data based on head motion is comparable to the usual data exclusion percentage [41]. 


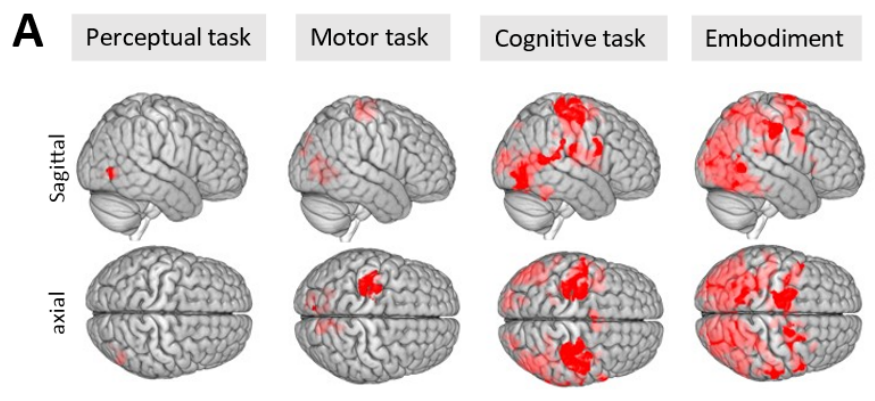

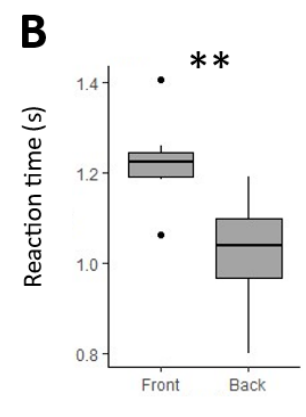

Character angle

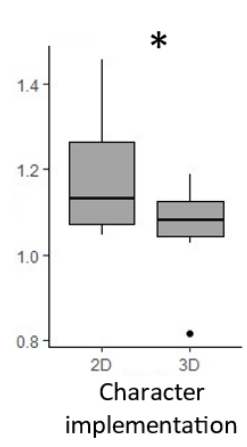

Figure 4: Brain activations of a typical subject (A) and cognitive task behavioral results (B). hMT+/V5 activation for random dot motion, dorsal motor cortex activations for arm movement, activation of temporo-parietal junction for OBT task, and parietofrontal regions activated by embodiment. We found both classical OBT effect (character angle) and a new effect (character implementation) using the virtual implementation of OBT task.
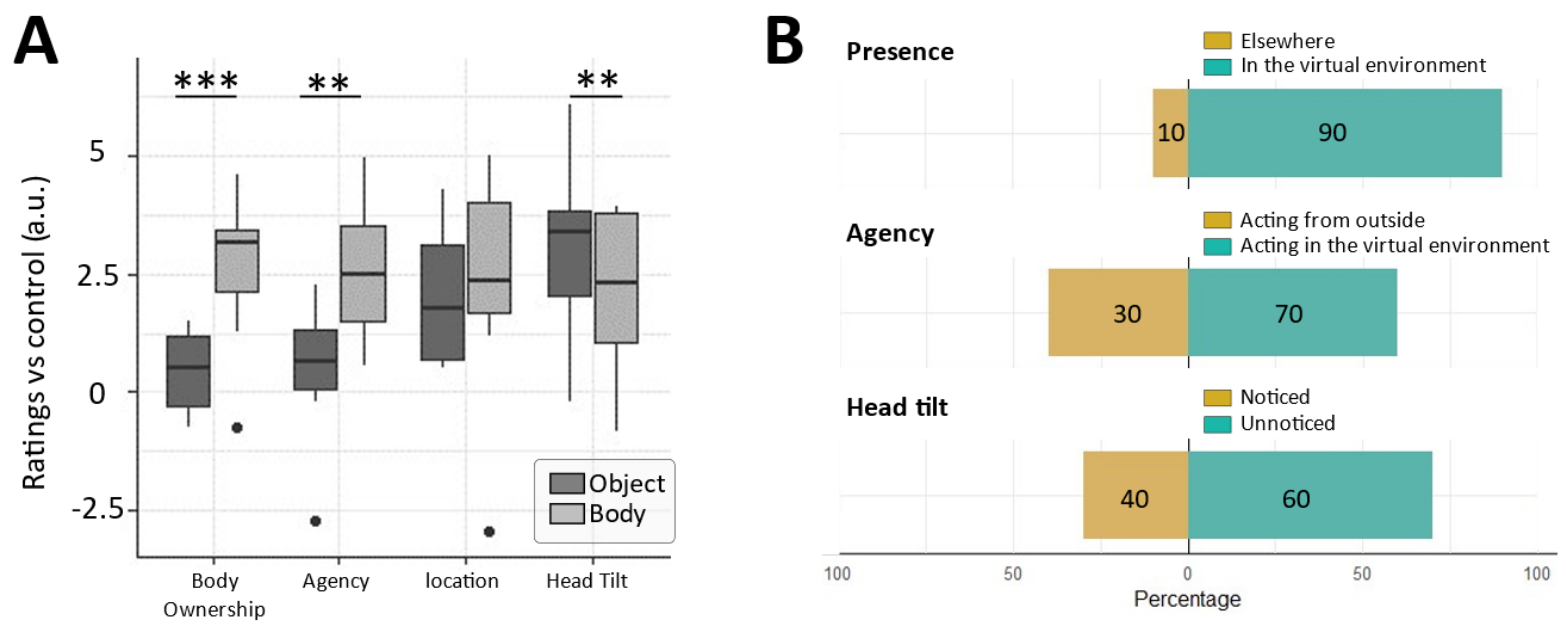

Figure 5: In-scanner (A) and post-experimental (B) questionnaire results. A. We observed a significant impact of the experience of body ownership, agency and head tilt noticing during the experiment. $B$. We observed high sense of presence, agency and low head tilt noticeability suggesting an overall good immersion all over the experiment. Boxplots lower and upper hinges correspond to the 25 th and 75 th percentiles, the black line is the median. Black dots are outlying values. ${ }^{*} \mathrm{p}<0.05$; ${ }^{* *} \mathrm{p}<0.01$; ${ }^{* * *} \mathbf{p}<\mathbf{0 . 0 0 1}$.

\section{DISCUSSION}

Our results show that the present VR-MRI platform is providing a fully controlled and lifelike virtual environment for neuroscience research. Using the present VR-MRI platform to present stimuli in immersive conditions (stereoscopic vision, large field of view, and sensorimotor interactions), we were able to confirm classical behavioral findings and brain activations observed in perceptual, motor, and cognitive tasks. Importantly, analyzing the MRI data, we show that the fMRI head motion-induced noise level was comparable to standard noise levels in MRI acquisition setups. Moreover, we showed that specific aspects of virtual embodiment were online modulated during task performance with the avatar and its animation. Specifically, the behavioral and neural correlates of the mental imagery involved in the own-body transformation task were modified by more immersive and ecological conditions (3D character perceived from a first-person embodied avatar). Finally, the analysis of fMRI data revealed activations in expected visual, motor, and cognitive regions in response to stimuli implemented in the virtual environment. With our pilot fMRI experiment, we demonstrated the feasibility of immersive VR involving interactions between virtual objects and motion-tracked upper limbs, in order to implement 

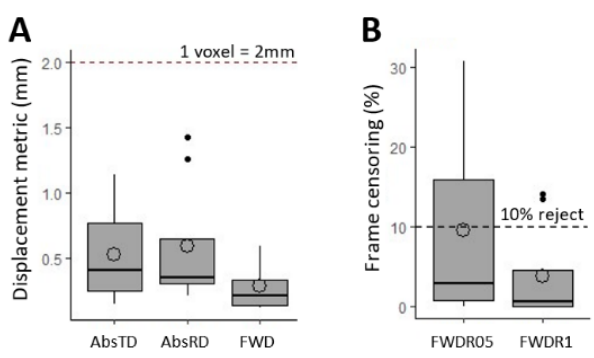

Figure 6: Participant-wise average fMRI head motioninduced noise metrics (A) and censoring estimates (B). Boxplots lower and upper hinges correspond to the 25th and 75th percentiles, black lines are median values and empty circles mean values. Black dots are outlying values.

ecological action-perception loops. Moreover, such design involving motor actions with more important body movements than in usual experimental designs provided sufficient signal-to-noise ratio for efficient fMRI analysis, validating our VR-MRI platform for neuroscience research. It also offers several opportunities for future neuroscience research.

\subsection{Opportunities enabled by the VR-MRI platform for neuroscience}

Beyond the confirmation of basic fMRI results, the virtualization of the experimental tasks opens numerous opportunities for neuroscience research.

First, the environment is providing the possibility of converting any cognitive neuroscience design already available as screen-based into an ecologically valid environment through virtual replicates of actual testing environments, but also to extend many known paradigms to a new fully virtual version. For instance, we replicated activation of motion-specific visual brain regions using a simple virtual screen displaying classical random-dot kinematograms. Moreover, the display of stimuli can be extended to fully virtual objects bringing literally another dimension to the study of perceptual processing of visual stimuli [32].

Second, this platform is also providing specific ecological relevance for sensorimotor processes: body movements are tracked in real time allowing coupling real body movements with virtual ones, going beyond the usual button-press toward more natural movement like pointing or lifting objects with accurate visual feedback. For instance, the study of fine gestures [9], tools use [17] or motor affordance [15] can be leveraged using the VR-MRI platform.

Third, we replicated the brain activation results of our visual, motor, and cognitive tasks using an immersive virtual version of the tasks. For instance, our more ecological version of a cognitive task (OBT) replicated the predicted behavioral and neural observations but also yielded unexpected changes that are worth further explorations (slowing down of reaction times for 3D characters instead of "flat" representations). The benefit of immersive VR has already been validated in other neuroscientific domains such as the study of memory. Recent evidence suggests that immersive virtual experience can lead to better autobiographical memory retrieval as compared to laboratory events based on flat-screen displays [22, 46], making our platform an essential tool to further extend this line of research.

Fourth, we enabled strong embodiment for the virtual avatar (manual interaction with virtual buttons induced a significant increase of body ownership and agency) enabling several manipulations linked to bodily self-consciousness $[5,8,34]$ such as the full-body illusion [24], body-swap illusions [35], or perspective illusions [19]. Crucially, we obtained those results while maintaining movement artifacts in the usually accepted range.

\subsection{Methodological recommendations and limitations}

As the platform was developed to provide the most immersive experience and ecologically valid scenarios compatible with fMRI, we identified several methodological aspects that are key to the success of a VR-MRI implementation.

First, immersing participants in a fully reproduced virtual depiction of the MRI room is important for several reasons. It is a reminder for participants of the situation in which they are lying on a scanner bed in a testing room and that they have to follow instructions and remain still. Conversely, immersing participants into an unrelated virtual space could have negative outcomes: they might forget about MRI constraints, get carried away and move too much (causing movement artifacts in MR signals), or even be abruptly reminded of their real location when trying to move the head or to stand up. The proposed entry and exit phases into and from the VR scenario are accordingly important to provide a gradual transition between reality and virtuality and, as such, deepen and fasten the immersion in VR [50]. Second, providing a firstperson view of the virtual avatar is key to a successful immersion of participants as numerous VR studies have shown [27, 45]. Importantly, the camera rotation that moves from looking up at the ceiling to a view compatible with looking down at one's body and feet, is essential for providing participants with an embodied view of the virtual environment. Interestingly, our data show that this viewpoint manipulation remained mostly unnoticed and is significantly more accepted when the avatar is shown. Habituation phases when participants observe how the virtual body moves according to their actual movements is equally important. In our pilot, we let the participant observe the movements of their knees and of their hands while holding tennis balls for a certain time. Holding a real object not only gives a scale to the virtual objects but ensures visuo-proprioceptive-tactile-motor coherence between the real and virtual hands in the absence of finger tracking. Further implementation of finger tracking could, however, simplify the matter entirely with benefits for research on manual interaction and fine motor control.

We know that breaks in presence [42], which typically occur when a participant is reminded of the outside physical reality during immersion, are highly detrimental to the overall VR experience and slow to recover from. It is thus important to provide all instructions inside the virtual environment itself and to avoid any reminders of the actual physical location (e.g., touch a keyboard in reality when there is none in virtuality). An experiment cannot, however, be done in one continuous block, as unexpected events during MRI 
experimentation cannot be entirely avoided (either for technical or human reasons). Small experimental runs of acquisitions with breaks have even been proven beneficial for performance in cognitive tasks and to reduce the amount of participant's head movement in the MRI [51], suggesting that separation of an experiment into multiple steps remain beneficial to an experiment. We thus implemented smooth transitions that interrupt the experimental flow without breaking immersion, with a 'checkpoint' mechanism to go back to a previous step while remaining in VR in case of a problem. This aspect could, however, benefit from visual improvements and should be more systematically evaluated by future research.

Overall, considering the complexity and the cost of the equipment of such a system, a limited implementation can also be considered if those principles are followed. For instance, a custom optical tracking system with fewer cameras to track color markers through the control window of the MRI room could suffice to minimally track a participant's body. A pre-recorded animation could also be used for the viewpoint manipulation, provided the synchrony with the MRI table movement is achieved in an alternate way. Similarly, simplified and generic 3D models of the MRI room and of the avatar could possibly be used if sufficient levels of presence and embodiment are validated.

\subsection{Outlook}

The implementation of the present VR-MRI platform in human neuroscience centers using fMRI will offer new opportunities for neuroscientists. It will not only allow testing experimental designs in a classical way using virtual screen display but extend it to immersive virtual implementation for perceptual, motor and cognitive tasks in simulated ecological conditions. For instance, much progress is ongoing in understanding spatial navigation and memory in humans using interactive immersive Virtual Reality inspired by the seminal discovery of grid cells in animals, a cell system acting like a "GPS in the brain" $[29,30]$. The potential advancements are not limited to the study of the spatial dimension. The study of social and affective processes, requiring interaction between the participant and other people "in person", would greatly benefit from the implementation of virtual interactions with realistic virtual animated characters. Conversely, research in VR engineering will benefit from the ability to directly study the brain mechanisms of the presence in virtual environment and of embodiment in virtual characters, with unprecedented possibilities. Overall, building experimental design going beyond screen display to ecological implementation would pave the way for fMRI experimentation in many fields of neuroscience. We could thus expect important progress in these fields thanks to a successful and rich implementation of virtual experimental tasks in a VR-MRI platform.

\section{ACKNOWLEDGMENTS}

This research was supported by the Bertarelli Foundation and the Foundation Campus Biotech Geneva (FCBG). We would like to specifically thank our colleagues Gilles Reymond for his assistance in the conception, Loan Mattera for her assistance during the MRI scans, Antoine Provost who reconstructed in 3D the Campus Biotech MRI room, Margaux Dubessy for her assistance in managing the creation of the MRI-compatible motion capture camera mount, for her help during the installation, and for the modeling and printing of some 3D pieces, Arthur Nguyen for his assistance in implementation of an early version of the validation protocol. We also would like to thank the FCBG and HEPIA teams for assistance in all MRI compatible mount systems creation and installation. We especially thank Sebastien Granon for his assistance in designing and building an Arduino-based MRI compatible tennis ball answer system, and for his assistance in building and installing inside the MRI room the MRI-compatible motion capture camera mount. Finally, we would like to thank the FCBG IT team for their support.

\section{REFERENCES}

[1] Shahar Arzy, Gregor Thut, Christine Mohr, Christoph M Michel, and Olaf Blanke. 2006. Neural basis of embodiment: distinct contributions of temporoparietal junction and extrastriate body area. The fournal of neuroscience: the official journal of the Society for Neuroscience 26, 31 (August 2006), 8074-81. DOI:https://doi.org/ 10.1523/JNEUROSCI.0745-06.2006

[2] Jane E. Aspell, Bigna Lenggenhager, and Olaf Blanke. 2009. Keeping in Touch with One's Self: Multisensory Mechanisms of Self-Consciousness. PLOS ONE 4, 8 (August 2009), e6488. DOI:https://doi.org/10.1371/journal.pone.0006488

[3] Domna Banakou, Raphaela Groten, and Mel Slater. 2013. Illusory ownership of a virtual child body causes overestimation of object sizes and implicit attitude changes. Proceedings of the National Academy of Sciences of the United States of America 110, 31 (July 2013), 12846-12851. DOI:https://doi.org/10.1073/pnas. 1306779110

[4] O. Blanke. 2005. Linking Out-of-Body Experience and Self Processing to Mental Own-Body Imagery at the Temporoparietal Junction. Fournal of Neuroscience 25, 3 (2005), 550-557. DOI:https://doi.org/10.1523/JNEUROSCI.2612-04.2005

[5] Olaf Blanke, Mel Slater, and Andrea Serino. 2015. Behavioral, Neural, and Computational Principles of Bodily Self-Consciousness. Neuron 88, 1 (2015), 145-166. DOI:https://doi.org/10.1016/j.neuron.2015.09.029

[6] Sidney Bovet, Henrique Galvan Debarba, Bruno Herbelin, Eray Molla, and Ronan Boulic. 2018. The Critical Role of Self-Contact for Embodiment in Virtual Reality. IEEE Transactions on Visualization and Computer Graphics 24, 4 (April 2018), 1428-1436. DOI:https://doi.org/10.1109/TVCG.2018.2794658

[7] F.C. Donders. 1969. On the speed of mental processes. Acta Psychologica 30, (January 1969), 412-431. DOI:https://doi.org/10.1016/0001-6918(69)90065-1

[8] Henrik H. Ehrsson. 2020. Chapter 8 - Multisensory processes in body ownership. In Multisensory Perception, K. Sathian and V.S. Ramachandran (eds.). Academic Press, 179-200. DOI:https://doi.org/10.1016/B978-0-12-812492-5.00008-5

[9] Michiel van Elk, Hein van Schie, and Harold Bekkering. 2014. Action semantics: A unifying conceptual framework for the selective use of multimodal and modalityspecific object knowledge. Physics of Life Reviews 11, 2 (June 2014), 220-250. DOI:https://doi.org/10.1016/j.plrev.2013.11.005

[10] Stephen A. Engel, David E. Rumelhart, Brian A. Wandell, Adrian T. Lee, Gary H. Glover, Eduardo-Jose Chichilnisky, and Michael N. Shadlen. 1994. fMRI of human visual cortex. Nature 369, 6481 (1994), 525-525. DOI:https://doi.org/10. 1038/369525a0

[11] M D Esposito, G K Aguirre, E Zarahn, D Ballard, R K Shin, and J Lease. 1998. Functional MRI studies of spatial and nonspatial working memory. (1998).

[12] Baptiste Gauthier, Lucie Bréchet, Florian Lance, Robin Mange, Bruno Herbelin, Nathan Faivre, Thomas A. W. Bolton, Dimitri Van De Ville, and Olaf Blanke. 2020. First-person body view modulates the neural substrates of episodic memory and autonoetic consciousness: A functional connectivity study. Neuroimage 223, (December 2020), 117370. DOI:https://doi.org/10.1016/j.neuroimage.2020.117370

[13] Beatrice de Gelder, Jari Kätsyri, and Aline W. de Borst. 2018. Virtual reality and the new psychophysics. British Journal of Psychology 109, 3 (2018), 421-426. DOI:https://doi.org/10.1111/bjop.12308

[14] R. Goebel, D. Khorram-Sefat, L. Muckli, H. Hacker, and W. Singer. 1998. The constructive nature of vision: direct evidence from functional magnetic resonance imaging studies of apparent motion and motion imagery. Eur 7 Neurosci 10, 5 (May 1998), 1563-1573. DOI:https://doi.org/10.1046/j.1460-9568.1998.00181.x

[15] J. Grèzes, M. Tucker, J. Armony, R. Ellis, and R. E. Passingham. 2003. Objects automatically potentiate action: an fMRI study of implicit processing. European Journal of Neuroscience 17, 12 (June 2003), 2735-2740. DOI:https://doi.org/10. 1046/j.1460-9568.2003.02695.x

[16] Bruno Herbelin, Roy Salomon, Andrea Serino, and Olaf Blanke. 2015. 5. Neural Mechanisms of Bodily Self-Consciousness and the Experience of Presence in Virtual Reality. In Human Computer Confluence. De Gruyter Open, Warsaw, Poland, 80-95. DOI:https://doi.org/10.1515/9783110471137-005

[17] J. Hermsdörfer, G. Terlinden, M. Mühlau, G. Goldenberg, and A.M. Wohlschläger. 2007. Neural representations of pantomimed and actual tool use: Evidence from an event-related fMRI study. NeuroImage 36, (January 2007), T109-T118. DOI:https: 
//doi.org/10.1016/j.neuroimage.2007.03.037

[18] Malte Husung and Eike Langbehn. 2019. Of Portals and Orbs: An Evaluation of Scene Transition Techniques for Virtual Reality. Proceedings of Mensch und Computer 2019, 245-254. Retrieved from https://doi.org/10.1145/3340764.3340779

[19] Silvio Ionta, Lukas Heydrich, Bigna Lenggenhager, Michael Mouthon, Eleonora Fornari, Dominique Chapuis, Roger Gassert, and Olaf Blanke. 2011. Multisensory Mechanisms in Temporo-Parietal Cortex Support Self-Location and First-Person Perspective. Neuron 70, 2 (2011), 363-374. DOI:https://doi.org/10.1016/j.neuron 2011.03.009

[20] Andreas Kalckert and H Ehrsson. 2012. Moving a Rubber Hand that Feels Like Your Own: A Dissociation of Ownership and Agency. Frontiers in Human Neuroscience 6, (2012), 40. DOI:https://doi.org/10.3389/fnhum.2012.00040

[21] Konstantina Kilteni, Raphaela Groten, and Mel Slater. 2012. The Sense of Embodiment in Virtual Reality. Presence: Teleoperators and Virtual Environments 21, 4 (November 2012), 373-387. DOI:https://doi.org/10.1162/PRES_a 00124

[22] Joanna Kisker, Thomas Gruber, and Benjamin Schöne. 2021. Virtual reality experiences promote autobiographical retrieval mechanisms: Electrophysiological correlates of laboratory and virtual experiences. Psychological Research 85, 7 (October 2021), 2485-2501. DOI:https://doi.org/10.1007/s00426-020-01417-x

[23] Nikolaus Kriegeskorte, Elia Formisano, Bettina Sorger, and Rainer Goebel. 2007 Individual faces elicit distinct response patterns in human anterior temporal cortex. Proc Natl Acad Sci USA 104, 51 (December 2007), 20600. DOI:https://doi org/10.1073/pnas.0705654104

[24] Bigna Lenggenhager, Tej Tadi, Thomas Metzinger, and Olaf Blanke. 2007. Video Ergo Sum: Manipulating BodilySelf-Consciousness. Science 317, 2007 (2007), 1096-1099. DOI:https://doi.org/10.1126/science.1143439

[25] Martin Lotze, Pedro Montoya, Michael Erb, Ernst Hülsmann, Herta Flor, Uwe Klose, Niels Birbaumer, and Wolfgang Grodd. 1999. Activation of Cortical and Cerebellar Motor Areas during Executed and Imagined Hand Movements: An fMRI Study. Journal of Cognitive Neuroscience 11, 5 (September 1999), 491-501. DOI:https://doi.org/10.1162/089892999563553

[26] Lara Maister, Mel Slater, Maria V. Sanchez-Vives, and Manos Tsakiris. 2015 Changing bodies changes minds: Owning another body affects social cognition. Trends in Cognitive Sciences 19, 6-12. DOI:https://doi.org/10.1016/j.tics.2014.11.001

[27] Antonella Maselli and Mel Slater. 2013. The building blocks of the full body ownership illusion. Front. Hum. Neurosci. 7, (2013). DOI:https://doi.org/10.3389/ fnhum.2013.00083

[28] Mara Mather, John T. Cacioppo, and Nancy Kanwisher. 2013. How fMRI Can Inform Cognitive Theories. Perspect Psychol Sci 8, 1 (January 2013), 108-113. DOI:https://doi.org/10.1177/1745691612469037

[29] Hyuk-June Moon, Baptiste Gauthier, Hyeong-Dong Park, Nathan Faivre, and Olaf Blanke. 2020. Sense of self impacts spatial navigation and hexadirectional coding in human entorhinal cortex. bioRxiv (January 2020), 2020.09.13.295246. DOI:https://doi.org/10.1101/2020.09.13.295246

[30] Edvard I Moser, Emilio Kropff, and May-Britt Moser. 2008. Place cells, grid cells and the brain's spatial representation system. Annual review of neuroscience 31, (January 2008), 69-89. DOI:https://doi.org/10.1146/annurev.neuro.31.061307. 090723

[31] Sebastian Oberdörfer, Martin Fischbach, and Marc Erich Latoschik. 2018. Effects of VE Transition Techniques on Presence, Illusion of Virtual Body Ownership, Efficiency, and Naturalness. Proceedings of the Symposium on Spatial User Interaction, 89-99. Retrieved from https://doi.org/10.1145/3267782.3267787

[32] Guy A. Orban. 2011. The Extraction of 3D Shape in the Visual System of Human and Nonhuman Primates. Annu. Rev. Neurosci. 34, 1 (July 2011), 361-388. DOI:https://doi.org/10.1146/annurev-neuro-061010-113819

[33] Sofia Adelaide Osimo, Rodrigo Pizarro, Bernhard Spanlang, and Mel Slater. 2015 Conversations between self and self as Sigmund Freud - A virtual body ownership paradigm for self counselling. Scientific Reports 5, 1 (September 2015), 1-14 DOI:https://doi.org/10.1038/srep13899

[34] Hyeong-Dong Park and Olaf Blanke. 2019. Coupling Inner and Outer Body for Self-Consciousness. Trends in Cognitive Sciences 23, 5 (May 2019), 377-388. DOI:https://doi.org/10.1016/j.tics.2019.02.002

[35] Valeria I. Petkova and H. Henrik Ehrsson. 2008. If I were you: Perceptual illusion of body swapping. PLoS ONE 3, 12 (2008). DOI:https://doi.org/10.1371/journal pone. 0003832

[36] Russell A. Poldrack. 2006. Can cognitive processes be inferred from neuroimaging data? Trends in Cognitive Sciences 10, 2 (February 2006), 59-63. DOI:https://doi. org/10.1016/j.tics.2005.12.004

[37] Thibault Porssut, Bruno Herbelin, and Ronan Boulic. 2019. Reconciling Being in-Control vs. Being Helped for the Execution of Complex Movements in VR. In 2019 IEEE Conference on Virtual Reality and 3D User Interfaces (VR), 529-537. DOI:https://doi.org/10.1109/VR.2019.8797716

[38] Jonathan D. Power, Anish Mitra, Timothy O. Laumann, Abraham Z. Snyder Bradley L. Schlaggar, and Steven E. Petersen. 2014. Methods to detect, characterize, and remove motion artifact in resting state fMRI. NeuroImage 84, (2014), 320-341. DOI:https://doi.org/10.1016/j.neuroimage.2013.08.048
[39] Maria V. Sanchez-Vives and Mel Slater. 2005. From presence to consciousness through virtual reality. Nature Reviews Neuroscience 6, 4 (April 2005), 332-339. DOI:https://doi.org/10.1038/nrn1651

[40] Lars Schwabe, Bigna Lenggenhager, and Olaf Blanke. 2009. The timing of temporoparietal and frontal activations during mental own body transformations from different visuospatial perspectives. Human Brain Mapping 30, 6 (2009), 1801-1812. DOI:https://doi.org/10.1002/hbm.20764

[41] Joshua S. Siegel, Jonathan D. Power, Joseph W. Dubis, Alecia C. Vogel, Jessica A. Church, Bradley L. Schlaggar, and Steven E. Petersen. 2014. Statistical improvements in functional magnetic resonance imaging analyses produced by censoring high-motion data points. Human Brain Mapping 35, 5 (May 2014), 1981-1996. DOI:https://doi.org/10.1002/hbm.22307

[42] Mel Slater. 2009. Place illusion and plausibility can lead to realistic behaviour in immersive virtual environments. Philos Trans R Soc Lond B Biol Sci 364, 1535 (December 2009), 3549-3557. DOI:https://doi.org/10.1098/rstb.2009.0138

[43] Mel Slater. 2018. Immersion and the illusion of presence in virtual reality. British fournal of Psychology 109, 3 (2018), 431-433. DOI:https://doi.org/10.1111/bjop. 12305

[44] Mel Slater and Maria V. Sanchez-Vives. 2016. Enhancing Our Lives with Immersive Virtual Reality. Frontiers in Robotics and AI 3, (2016), 74. DOI:https://doi.org/10. 3389/frobt.2016.00074

[45] Mel Slater, Bernhard Spanlang, Maria V. Sanchez-Vives, and Olaf Blanke. 2010. First person experience of body transfer in virtual reality. PLoS One 5, 5 (May 2010), e10564. DOI:https://doi.org/10.1371/journal.pone.0010564

[46] S. Adam Smith. 2019. Virtual reality in episodic memory research: A review. Psychonomic Bulletin \& Review 26, 4 (August 2019), 1213-1237. DOI:https://doi. org/10.3758/s13423-019-01605-w

[47] William Steptoe, Anthony Steed, and Mel Slater. 2013. Human tails: Ownership and control of extended humanoid avatars. IEEE Transactions on Visualization and Computer Graphics 19, 4 (April 2013), 583-590. DOI:https://doi.org/10.1109/ TVCG.2013.32

[48] Pawel Tacikowski, Marieke L. Weijs, and H. Henrik Ehrsson. 2020. Perception of Our Own Body Influences Self-Concept and Self-Incoherence Impairs Episodic Memory. iScience 23, 9 (September 2020), 101429. DOI:https://doi.org/10.1016/j. isci.2020.101429

[49] Martin Usoh, Ernest Catena, Sima Arman, and Mel Slater. 2000. Using presence questionnaires in reality. Presence: Teleoperators and Virtual Environments 9, 5 (2000), 497-503. DOI:https://doi.org/10.1162/105474600566989

[50] Dimitar Valkov and Steffen Flagge. 2017. Smooth immersion: the benefits of making the transition to virtual environments a continuous process. In Proceedings of the 5th Symposium on Spatial User Interaction (SUI '17), Association for Computing Machinery, Brighton, United Kingdom, 12-19. DOI:https: //doi.org/10.1145/3131277.3132183

[51] T. Waltemate, D. Gall, D. Roth, M. Botsch, and M. E. Latoschik. 2018. The Impact of Avatar Personalization and Immersion on Virtual Body Ownership, Presence, and Emotional Response. IEEE Transactions on Visualization and Computer Graphics 24, 4 (April 2018), 1643-1652. DOI:https://doi.org/10.1109/TVCG.2018.2794629

[52] Jeff Zacks, Bart Rypma, J.D.E Gabrieli, Barbara Tversky, and Gary H Glover. 1999. Imagined transformations of bodies: an fMRI investigation. Neuropsychologia 37, 9 (August 1999), 1029-1040. DOI:https://doi.org/10.1016/S0028-3932(99)00012-3

\section{APPENDICES}

\section{A HARDWARE SCHEMATICS \\ B STEREOSCOPIC ACUITY TEST}

To evaluate if participants have stereoscopic vision, we presented them with a stereoscopic acuity test in a VR headset (Oculus Go). Random dot stereograms were displayed in which a centered box appeared either in front of or behind the rest of the image. Participants were asked to respond in a three-alternative forced-choice task ("Front"; "Behind"; "I do not know"). A maximum of 14 trials was presented with 10 levels of increasing difficulty as stereo disparity decreases (random dot stereograms were pictures of 1024x768px occupying $43.6^{\circ}$ field of view horizontally, with disparity applied on a square of $380 \times 380 \mathrm{px}$, one horizontal voxel depth per level, the minimum disparity is 1 at level 10). When a correct answer was given, the difficulty increased by 1 , when an incorrect answer is given, the difficulty decreased by 1 . The test stopped when the participant reached the maximum of 14 trials or when the participant failed three times at the same difficulty. 


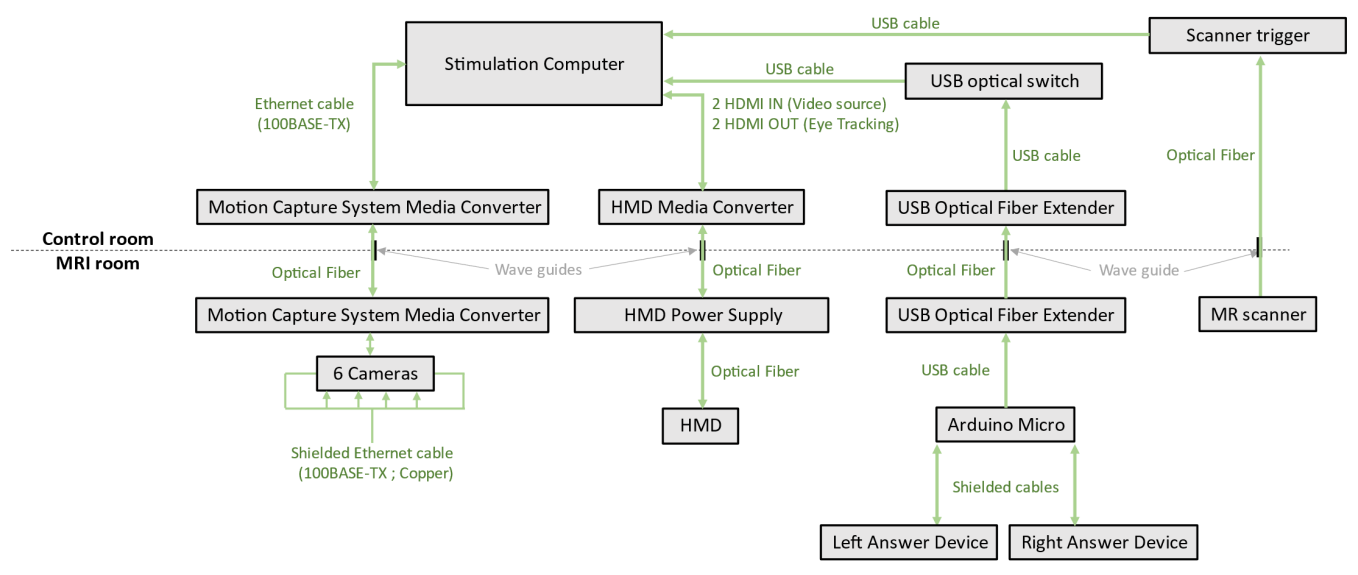

Figure 7: Functional schematic of the hardware connections between the MRI scanner room and the control room.

\section{MRI SCANNING AND ANALYSIS PARAMETERS}

MR images were acquired using a 3T MRI scanner (MAGNETOM PRISMA; Siemens) using a 64-channel head coil. Each participant underwent $5.21 \mathrm{~min}$ anatomical imaging using a T1-weighted MPRAGE sequence (192 slices; slice thickness $=0.90 \mathrm{~mm}$; repetition time $=2300 \mathrm{~ms}$; echo time $=2.32 \mathrm{~ms}$; flip angle $=8^{\circ}$; voxel size $=0.9 \times 0.9 \times 0.9 \mathrm{~mm} 3 ; \mathrm{FOV}=240 \mathrm{~mm}$; GRAPPA factor $=2$ ). They also underwent four 10-minutes (two blocks of each of the VR tasks: 2D- and 3D-OBT) and one 5-minute sessions (Block of VR tasks: RDK, MVT and also eyes-open resting-state) of functional imaging with blood oxygenation level-dependent contrast using a gradient-echo echo-planar imaging sequence (75 slices; slice thickness $=2 \mathrm{~mm}$; repetition time $=1250 \mathrm{~ms}$; echo time $=32 \mathrm{~ms}$; voxel size $=2 \times 2 \times 2 \mathrm{~mm} 3$; FOV $=224 \mathrm{~mm}$; Multiband factor: 5). Familiarization, entry, and exit phases lasted in total and on average 4.4 min and no image was acquired in those sessions.

We used statistical parametric mapping (SPM12) for images preprocessing with slice timing correction, realignment, coregistration with the structural image, normalization to Montreal Neurological Institute stereotactic space, and spatial smoothing with a $5 \mathrm{~mm}$ full-width half-maximum isotropic Gaussian kernel. General linear models were estimated subject-wise based on a design matrix that covered all five fMRI sessions and included regressors for every experimental condition of the paradigm (convolved with a canonical hemodynamic response function) as well as nuisance covariates from the motion parameters and session blocks. A high-pass filter (128 s cutoff) was applied to remove slow drifts unrelated to the paradigm 